\title{
A ENFERMAGEM DOS CENTROS DE ATENÇÃO PSICOSSOCIAL DE UMA CAPITAL DO NORDESTE DO BRASIL
}

\author{
THE NURSING OF PSYCHOSOCIAL CARE CENTERS IN A NORTHEAST \\ CAPITAL OF BRAZIL
}

\section{LA ENFERMERÍA DE LOS CENTROS DE ATENCIÓN PSICOSOCIAL DE UN CAPITAL DEL NORDESTE DE BRASIL}

\author{
John Victor dos Santos Silva ${ }^{1}$, Thyara Maia Brandão ${ }^{2}$
}

\begin{abstract}
RESUMO
Objetivo: descrever as características da enfermagem que atua nos Centros de Atenção Psicossocial de uma capital do nordeste do Brasil. Método: Trata-se de um estudo descritivo, de abordagem quantitativa, realizado em cinco CAPS de Maceió-AL, tendo como sujeitos os trabalhadores de enfermagem. Foi aplicado questionário estruturado e os dados agrupados e analisados por estatística simples, por meio de porcentagem. Resultados: Dos 41 trabalhadores de enfermagem que participaram do presente estudo, 12 exerciam o cargo de auxiliar de enfermagem; 11 exerciam o cargo de técnico de enfermagem e 18, o cargo de enfermeiro. Destes, $88 \%$ eram mulheres e $12 \%$ homens. O estudo ainda traz informações referentes à formação, trabalho, capacitação e atividades desempenhadas por esses profissionais. Conclusão: Percebeu-se que os profissionais possuem características específicas e desempenham funções primordiais na realização das ações nos Centros de Atenção Psicossocial de Maceió.
\end{abstract}

Descritores: Enfermagem; Saúde Mental; Serviços de Saúde Mental.

\begin{abstract}
Objective: to describe the characteristics of nursing that works in the Psychosocial Care Centers of a capital city in northeastern Brazil. Methodology: This is a descriptive study with a quantitative approach. The study was carried out in five CAPS in Maceió-AL and the subjects were nursing workers. A structured questionnaire and the grouped data were applied and analyzed by simple statistics, by percentage. Results: Of the 41 nursing workers who participated in the present study, 12 were nursing auxiliaries; 11 were in charge of nursing technicians; and 18 the position of nurses. Of these, 88declared themselves women and 12\% declared themselves men. The study still provides information regarding the training, job training, and activities performed by these professionals. Conclusion: It was perceived that the professionals have specific characteristics and perform the primary functions of the actions in the Centers of Psychosocial Attention of Maceió.
\end{abstract}

Keywords: Nursing; Mental Health; Mental Health Services.

\footnotetext{
1 Acadêmico de Enfermagem pela Universidade Estadual de Ciências da Saúde de Alagoas (UNCISAL). Presidente da Liga Acadêmica Interdisciplinar de Saúde Mental (LAISME-UNCISAL).

${ }^{2}$ Enfermeira. Mestre em Enfermagem. Especialista em Saúde Mental e Psiquiatria. Professora da Universidade Estadual de Ciências da Saúde de Alagoas (UNCISAL). Tutora da Liga Acadêmica Interdisciplinar de Saúde Mental (LAISME-UNCISAL).
} 


\section{RESUMEN}

Objetivo: describir las características de la enfermería que actúa en los Centros de Atención Psicosocial de una capital del nordeste de Brasil. Metodología: Se trata de un estudio descriptivo de abordaje cuantitativo. El estudio ocurrió en cinco CAPS de Maceió-AL y los sujetos fueron los trabajadores de enfermería. Se aplicó cuestionario estructurado y los datos agrupados y analizado por estadística simple, a través de porcentaje. Resultados: De los 41 trabajadores de enfermería que participaron del presente estudio, 12 ejercían el cargo de auxiliares de enfermería; 11 ejercían el cargo de técnicos de enfermería; y 18 el cargo de enfermeros. De ellos, el $88 \%$ se declararon mujeres y el 12\% se declararon hombres. El estudio aún trascende informaciones referentes a la formación, trabajo capacitación, y actividades desempeñadas por esos profesionales. Conclusión: Se percibió que los profesionales poseen características específicas y desempeñan funciones primordiales la realización de las acciones en los Centros de Atención Psicosocial de Maceió

Descriptores: Enfermería; Salud Mental; Servicios de Salud Mental.

\section{INTRODUÇÃO}

A enfermagem é uma profissão que tem como característica o cuidado com a saúde dos indivíduos, família e comunidade. Ela está presente em todas as fases e situações da vida, considerando que esta tem um valor imensurável. ${ }^{1}$ Ela está presente também junto aos momentos nos quais as pessoas enfrentam extremas situações de sofrimento mental, sejam nos domicílios ou nos serviços de saúde. ${ }^{2}$

Por muitos anos, a assistência de enfermagem à pessoa em sofrimento mental foi determinada pelos hospitais psiquiátricos, desempenhando uma função bastante característica à época, que era de auxiliar os médicos psiquiatras nos procedimentos e tratamentos, além de realizar a administração de medicamentos, de vigilância e controle dos pacientes, permanecendo assim por muitos anos. ${ }^{3,4}$

Com as modificações das políticas de saúde mental e o surgimento de novos serviços, como os Centros de Atenção Psicossocial, a enfermagem se viu também tendo que repensar suas práticas, incluindo estratégias que fossem além das usualmente exercidas, de modo a contribuir com o novo modelo de cuidado, voltado para inserção dos indivíduos em sofrimento mental na família, na comunidade e no trabalho. ${ }^{1}$

O principal serviço de saúde mental existente no Sistema Único de Saúde (SUS) é o Centro de Atenção Psicossocial (CAPS). Estes são serviços de saúde mental que contam com uma equipe especializada para prestar assistência às pessoas em sofrimento mental. Surgiram durante o Processo da Reforma Psiquiátrica e realizam atividades que contribuem para o processo de reabilitação psicossocial desses indivíduos. ${ }^{5}$

Os profissionais de Enfermagem, em nível médio e superior, compõem a equipe mínima para o trabalho nos CAPS em todas as suas 07 modalidades existentes:

CAPS I, CAPS II, CAPS III, CAPS i II, 
CAPS ad II, CAPS ad III e CAPS ad IV. Esses profissionais são uma exigência do Ministério da Saúde para desempenhar papéis e contribuir significativamente na assistência em saúde mental no Brasil. ${ }^{6}$

Os Enfermeiros, Técnicos ou Auxiliares de Enfermagem compõem o quadro de profissionais que atuam nos CAPS e desempenham atividades específicas, como a Consulta de Enfermagem, a Sistematização da Assistência de Enfermagem, Administração de Medicamentos, Realização de Curativos, entre outros, que são de competência desses nos serviços de saúde, inclusive nos Centros de Atenção.

Além disso, desenvolvem outras atividades em conjunto com os demais profissionais do serviço, como as visitas domiciliares, oficinas terapêuticas, acolhimento e atividades fora dos serviços com os usuários, conferindo, também, um trabalho de caráter interdisciplinar, que é um ponto forte dos CAPS, que no Brasil teve crescimento considerável, da mesma forma que o número de profissionais de enfermagem atuando nesses serviços. ${ }^{7,8}$

Com todas as mudanças que existiram ao longo dos anos no processo do cuidar em saúde mental, a enfermagem percorreu essa trajetória e ainda permanece atuante, o que nos leva a perceber a importância do fazer desses profissionais. ${ }^{2}$ Assim, o presente estudo tem como objetivo descrever as características sobre a formação, aspectos do trabalho e atividades desenvolvidas pelos profissionais de enfermagem que estão nos Centros de Atenção psicossocial de uma capital do nordeste do Brasil.

\section{MÉTODO}

Trata-se de um estudo descritivo com abordagem quantitativa. Os estudos descritivos são os mais indicados para pesquisas que têm como finalidade trazer características ou traçar perfis dos indivíduos, verificando a relação entre as variáveis nas abordagens quantitativas. ${ }^{9}$

O estudo aconteceu em cinco CAPS de uma capital do nordeste do Brasil, vinculados à Secretaria Municipal de Saúde. Os sujeitos da pesquisa foram os profissionais de enfermagem, compreendendo uma população de 56 trabalhadores. O critério de inclusão estabelecido foi fazer parte da equipe de enfermagem dos CAPS e os critérios de exclusão adotados foram estar de licença maternidade, licença médica ou de férias.

Para esta pesquisa foi estabelecida a amostra censitária, que é escolhida quando se quer investigar toda a população ou um universo em específico. ${ }^{10}$ Assim, participaram 41 do total de 56 profissionais de enfermagem que atuam nos CAPS do município, onde os 15 restantes não entraram na amostra, pois se enquadraram 
nos critérios de exclusão (estar de férias e licença maternidade ou médica) ou por não desejarem participar do estudo. Os profissionais foram convidados a participar da pesquisa nos próprios CAPS em que trabalhavam, por meio de um convite verbal, onde o pesquisador forneceu as informações necessárias quanto à realização do estudo e suas etapas.

O Termo de Consentimento Livre e Esclarecido foi entregue para coleta das assinaturas, baseado nas diretrizes da resolução CNS/MS 466/12. O presente estudo foi submetido ao Comitê de Ética em Pesquisa e teve sua aprovação através do CAAE: 84827417.3.0000.5011.

A coleta de dados aconteceu nos meses de julho e agosto de 2018, tendo sido utilizados três questionários estruturados específicos, elaborados pelos autores, sendo um para cada categoria profissional de enfermagem dos CAPS (Enfermeiro,
Técnico e Auxiliar de Enfermagem). Não foi aplicado teste prévio do questionário nem avaliação por pares. Cada questionário continham perguntas referentes a informações sociodemográficas, de formação profissional, aspectos do trabalho e atividades desenvolvidas. Os dados foram organizados e analisados descritivamente pela estatística simples, através de porcentagens.

\section{RESULTADOS}

Dos 41 trabalhadores de enfermagem que participaram do presente estudo, 12 exerciam o cargo de auxiliar de enfermagem; 11 exerciam o cargo de técnico de enfermagem; e 18 o cargo de enfermeiro, como está descrito na tabela 1. Destes, 88\% (36 profissionais) declararamse mulheres e $12 \%$ (05 profissionais) declararam-se homens.

Tabela 1 - Número e proporção dos trabalhadores de enfermagem dos CAPS por categoria profissional em exercício na SMS. Brasil, 2018.

\begin{tabular}{lcc}
\hline Categoria Profissional & n & \% \\
\hline Auxiliar de Enfermagem & 12 & 29 \\
Técnico de Enfermagem & 11 & 27 \\
Enfermeiro & 18 & 44 \\
\hline Total & 41 & 100 \\
\hline
\end{tabular}

Fonte: Dados da pesquisa, 2018.

Todos os trabalhadores possuem nacionalidade brasileira, sendo $44 \%$ (18) naturais da capital, 29\% (12) de outros municípios do estado; $17 \%$ (07) de outros estados nordestinos; e 10\% (04) naturais de outras regiões do país. Destes, 85\% (35) vivem na capital; $12 \%$ (05) vivem em outros municípios do estado e $2 \%$ (01) em outro estado nordestino. 
Sobre o tempo de formação dos trabalhadores, em suas respectivas categorias, em exercício nos CAPS, 90\% (37) formaram-se há mais de 05 anos; 5\% (02) formaram-se há 04 anos; 2\% (01) há 05 anos e 2\% (01) há menos de 01 ano.

Ainda com relação à formação, $54 \%$ (22) são formados em outro curso de nível superior; 44\% (18) não possuem outro tipo de formação e $2 \%$ (01) preferiu não responder. Dos que possuíam outra formação, 73\% (16) são auxiliares ou técnicos de enfermagem e $27 \%$ (06) são enfermeiros. Destes, $50 \%$ (11) possuem formação em outro curso da área da saúde; $36 \%$ (08) outro curso na área de humanas; 5\% (01) com duas formações, na área de saúde e humanas; e 9\% (02) preferiram não responder.

Foi constatado que $51 \%$ (21) não participam de programa de educação permanente em saúde; $37 \% \quad$ (15) participam; e $12 \%$ (05) preferiram não responder.

A maioria dos profissionais de enfermagem pesquisados nunca trabalhou em Hospitais Psiquiátricos, sendo representado por $88 \%$ (36). Apenas $12 \%$ (05) já trabalharam nos serviços institucionais de psiquiatria.

Com relação ao tipo de vínculo empregatício com os CAPS, todos trabalham em regime de 30 horas semanais, com $80 \%$ (33) sendo servidores efetivos, estatutários e $20 \% \quad(08)$ servidores temporários, em regime de contratação. Dos trabalhadores com vínculo temporário, todos são enfermeiros.

Quando analisado o tempo de trabalho, o resultado foi bastante variado. A maioria dos trabalhadores, $41 \%$ (17), estão nos CAPS há mais de 05 anos; 20\% (08) estão no CAPS há 04 anos; 20\% (08) há 03 anos; $7 \%$ (03) estão há 05 anos; 5\% (02) há 02 anos; $5 \%$ (02) há 06 meses e 2\% (01) há 01 ano.

Quando perguntados sobre a decisão de trabalhar nos CAPS, 51\% (21) responderam que escolheram pelo serviço; $34 \%$ (14) relataram que não escolheram pelo serviço e $15 \%$ (06) preferiram não responder.

Sobre o inicio das atividades nos serviços, $73 \%$ (30) relataram que não receberam nenhum treinamento para trabalhar nos CAPS; $20 \%$ (08) receberam ao menos um treinamento e $7 \%$ (03) não quiseram responder.

Referente à frequência de treinamentos e capacitação profissional recebida pela enfermagem para o trabalho nos CAPS, as respostas foram bastante diversificadas. Uma parcela dos trabalhadores não recebeu nenhum tipo de treinamento desde quando ingressaram nos serviços, e uma boa parte preferiu não responder a essa questão, como mostra a tabela 2. 
Tabela 2 - Frequência de Treinamento e Capacitação Profissional recebida pela Enfermagem para desempenhar as atividades nos CAPS. Brasil, 2018.

\begin{tabular}{lcc}
\hline Frequência de Treinamento e Capacitação Profissional & n & \% \\
\hline Anualmente & 8 & 20 \\
Semestralmente & 1 & 2 \\
Trimestralmente & 1 & 2 \\
Mensalmente & 3 & 7 \\
Semanalmente & 1 & 2 \\
Não recebi desde que comecei a trabalhar no CAPS & 13 & 32 \\
Não desejo responder & 14 & 34 \\
\hline Total & 41 & 100 \\
\hline
\end{tabular}

Fonte: Dados da pesquisa, 2018.

Sobre o estímulo para capacitação no trabalho, $66 \%$ (27) informaram que não recebem incentivos para se capacitar na área; $15 \%$ (06) disseram que recebem incentivos; 20\% (08) preferiram não responder à pergunta.
As atividades mais desempenhadas pelas equipes de enfermagem dos CAPS estão descritas no quadro 1 e mostram as respostas que apareceram nos questionários de todos os entrevistados.

Quadro 1 - Atividades mais realizadas pela equipe de enfermagem e pelos enfermeiros dos CAPS. Brasil, 2018.

\begin{tabular}{|c|c|}
\hline Atividades da Equipe de enfermagem & Atividades dos Enfermeiros \\
\hline Administração de Medicamentos & Supervisão da equipe de enfermagem \\
\hline Curativos simples & Consulta de enfermagem \\
\hline Medidas antropométricas & Grupos Terapêuticos \\
\hline Verificação dos sinais vitais & Visitas Domiciliares \\
\hline Exame de glicemia capilar (HGT) & Projeto Terapêutico Singular \\
\hline $\begin{array}{c}\text { Sistematização da Assistência de } \\
\text { Enfermagem (SAE) }\end{array}$ & Educação em Saúde \\
\hline
\end{tabular}

Fonte: Dados da pesquisa, 2018.

Quanto perguntados sobre gostar do trabalho, $76 \%$ (31) responderam que gostam muito do trabalho; 20\% (08) disseram que não gostam muito; $2 \%(01)$ relatou que gosta do trabalho; e 2\% (01) disseram que não gostam do trabalho.

$$
\text { Com relação a se desligar do }
$$
trabalho nos CAPS; 56\% (23) não pensam em se desligar; 37\% (15) às vezes sentem vontade de se desligar; 5\% (02) sentem vontade de se desligar dos CAPS e $2 \%$ (01) preferiu não responder.

\section{DISCUSSÃO}

É possível encontrar mais profissionais de enfermagem mulheres do 
que homens nos serviços de saúde, a exemplo dos CAPS. Uma pesquisa realizada através do convênio do Conselho Federal de Enfermagem (COFEN) e a Fundação Osvaldo Cruz (Fiocruz) sobre o perfil da enfermagem no Brasil informa que no estado de Alagoas $88 \%$ dos profissionais são mulheres, corroborando a proporção deste estudo, no qual $88 \%$ dos trabalhadores de enfermagem dos CAPS da capital são mulheres. ${ }^{11}$

Essa presença expressiva de mulheres na profissão é história, por ser a enfermagem considerada uma ocupação realizada inicialmente pelas freiras em serviços de caridades para os pobres e excluídos da sociedade, o que levou à feminização da profissão, que até anos atrás era exercida exclusivamente por mulheres. Isso vem mudando recentemente, dada a necessidade e exigências do mercado de trabalho. ${ }^{12}$

Uma pesquisa organizada por Machado e colaboradores ${ }^{11}$ relata que $75 \%$ dos profissionais são naturais do estado onde o estudo foi realizado, o que se assemelha com os $73 \%$ dos profissionais de enfermagem dos CAPS do município que também são naturais do estado. Já sobre o tempo de formação, o estudo mostra que a maioria dos profissionais de enfermagem possui sua formação, seja do nível superior ou médio-técnico, há mais de cinco anos, também corroborando esse estudo.
A maioria dos profissionais de enfermagem não participa de programas de educação permanente em saúde. Isso se deve a inúmeros fatores; contudo, depende principalmente de iniciativa das respectivas Secretarias Municipais e Estaduais em fornecer e proporcionar oportunidades para os trabalhadores. ${ }^{13}$

Os CAPS são serviços de saúde relativamente novos e que foram iniciados em meados de 2000, permanecendo em expansão pelo território brasileiro, o que implica que boa parte dos profissionais de enfermagem que tenham prestado assistência às pessoas em sofrimento mental tenha trabalhado em Hospitais Psiquiátricos durante o período inicial da Reforma Psiquiátrica. Os cursos mais recentes já estão formando os profissionais de enfermagem para o trabalho nos serviços substitutivos. ${ }^{14}$

Os CAPS são serviços de saúde pública e que necessitam de servidores públicos para desenvolver as atividades propostas. Quando o quadro de trabalhadores efetivos não é suficiente para o funcionamento, os municípios podem fazer contratação ou seleção para aquisição de recursos humanos para desempenhar papéis específicos nos serviços de saúde, o que é permitido pelo SUS. ${ }^{15}$ Porém, o fato de haver profissionais que prestam serviços na saúde pública de forma temporária faz aumentar a rotatividade de trabalhadores 
nos serviços. A enfermagem é considerada, em alguns estudos, como a categoria profissional que mais sofre rotatividades nos CAPS da maioria das capitais, o que justifica o pouco tempo de trabalho de metade dos profissionais deste estudo. ${ }^{16}$

A área da saúde mental, em especial da Atenção Psicossocial, é muito específica e que tem pouca procura pelos profissionais de enfermagem, talvez pela complexidade do trabalho ou por falta de interesse pessoal do profissional. Para trabalhar na saúde mental é preciso pelo menos um pouco de afinidade com o processo de trabalho, mas alguns estudos mostram que não é isso o que acontece. Muitos profissionais acabam indo trabalhar nos serviços de saúde mental, a exemplo dos CAPS, por ser a oportunidade que apareceu no momento da inserção do mercado de trabalho. ${ }^{17}$

A capacitação para o trabalho nos CAPS merece atenção, pois não é algo apenas exclusivo dos profissionais de enfermagem. A maioria dos trabalhadores inseridos nos serviços de saúde mental não recebe treinamento ou capacitação para iniciar suas atribuições nos dispositivos. Isso impacta consideravelmente nos resultados do processo de reabilitação psicossocial que os CAPS preconizam. A assistência direta para as pessoas em sofrimento mental fica bastante prejudicada. A formação durante a graduação muitas vezes não é suficiente para que os trabalhadores compreendam o fazer na saúde mental. Existem algumas profissões que se aproximam mais durante a formação sobre as questões que norteiam a atenção psicossocial, já outras profissões acabam tendo que aprender durante o próprio trabalho no serviço. ${ }^{5,18}$

A pouca formação para o trabalho na saúde mental causa certa angústia nos profissionais e isso muitas vezes gera um sentimento de frustação, contribuindo também para o cansaço no trabalho. No entanto, isso não é suficiente para que os trabalhadores queiram se desligar completamente dos CAPS, como mostra a presente pesquisa, na qual mais da metade dos profissionais não pensam em sair do serviço. ${ }^{8}$

É comum que os auxiliares e técnicos de enfermagem possuam formação em nível superior. A maioria procura formação na área de saúde e biológicas, realizando, muitas vezes, o próprio curso de graduação em enfermagem ou até mesmo buscando outra formação profissional. Alguns possuem formação na área de humanas, se afastando da área da saúde. O que leva os auxiliares e técnicos de enfermagem buscar a graduação em nível superior é uma melhor colocação no mercado de trabalho; porém, muitos deles conciliam trabalhos em ambas as formações, atuando como profissional de nível médio em enfermagem, ou como 
enfermeiros e até mesmo profissionais de outras categorias da saúde e áreas distintas. $^{12}$

Os profissionais de enfermagem desempenham atribuições muito características da profissão, como administração de medicamentos, curativos, verificação dos sinais vitais, exame de glicemia capilar, além das visitas domiciliares e participação nos grupos e oficinas terapêuticas. Aos enfermeiros são destinadas as consultas de enfermagem, realização do acolhimento, construção do PTS do usuário do serviço, educação em saúde, elaboração das oficinas e grupos terapêuticos, dentre outros. Embora os enfermeiros se ocupem de atribuições específicas de enfermagem, a maioria desenvolve atividades importantes no processo de reabilitação psicossocial dos usuários dos CAPS, seja em maior ou menor frequência. ${ }^{19}$

Os enfermeiros também possuem papel importante nos grupos e oficinas terapêuticas realizadas pelos CAPS. Boa parte desses profissionais não apenas participa dessas atividades como também ajuda na construção, acontecendo preferencialmente de forma coletiva, com profissionais de outras formações de nível superior; mas a enfermagem também cria grupos com os usuários dos CAPS. ${ }^{20}$

Outra atividade bastante realizada nos CAPS é a construção dos PTS dos usuários, em que os enfermeiros também contribuem. O PTS que é aplicado nos CAPS é um conjunto de ações terapêuticas a serem desenvolvidas pelos usuários ao longo da semana e que visam proporcionar a reabilitação psicossocial desses sujeitos. A construção é uma atividade realizada pelos profissionais de nível superior, mas nada impede que os demais profissionais da equipe de enfermagem possam colaborar. ${ }^{14}$

A utilização da Sistematização da Assistência de Enfermagem e a Consulta de enfermagem nos CAPS são atividades de utilização pouco frequentes; porém, quando utilizadas pelos enfermeiros, promovem organização nas atividades da equipe de enfermagem e também aumentam as contribuições para a saúde integral dos sujeitos para além do esperado nas atividades convencionais. ${ }^{16}$

Trabalhar a consulta de enfermagem nos CAPS é um desafio para a maioria dos enfermeiros. Muitos serviços não dispõem de salas reservadas e quando elas existem, são divididas com a maioria dos profissionais onde são realizados os grupos terapêuticos, o que acaba concorrendo com as consultas dos médicos, psicólogos e terapeutas. Quando o enfermeiro não se apropria dessa atividade e mostra a sua importância para a equipe multidisciplinar, as consultas acabam por ficar remetidas apenas aos acolhimentos, que não 
compreende a realidade da consulta de enfermagem. $^{7}$

Além dessas atividades apresentadas, não podemos esquecer as visitas domiciliares, que são oportunidades para que os profissionais possam acompanhar e conhecer a realidade dos usuários dos CAPS. As visitas domiciliares são mais utilizadas pelas equipes da Estratégia Saúde da Família, mas também foram bastante incorporadas no processo de trabalho dos CAPS, uma vez que boa parte dos usuários não possui autonomia suficiente para irem até o serviço, muitos são trazidos pelos carros da secretaria de saúde. Quando alguns desses usuários têm alguma debilidade ou são impossibilitados de saírem de suas casas, as equipes se organizam e se revezam para realizarem atendimento nos domicílios, o que promove uma maior integralidade e universalidade dos serviços. ${ }^{8}$

Todas essas questões que norteiam as características da enfermagem e as suas atribuições nos CAPS ajudam a compreender melhor o processo de trabalho nesses serviços, principalmente por parte da equipe de enfermagem, que são profissionais importantes para o cuidado das pessoas em sofrimento mental. Entretanto, é preciso se aprofundar em questões subjetivas que envolvem o processo de trabalho da enfermagem em saúde mental para conhecer se as informações aqui apresentadas interferem de forma positiva ou negativa no processo de reabilitação psicossocial dos usuários dos serviços. ${ }^{3}$

As principais limitações do estudo são referentes ao seu recorte temporal e geográfico, podendo não ser referência para os profissionais de enfermagem das demais capitais do nordeste e do Brasil. A amostra do estudo, apesar de ser censitária, não compreendeu o número mínimo de participantes para ser aplicado algum teste estatístico de modo a servir de referência.

\section{CONCLUSÃO}

A presente pesquisa buscou identificar as características da enfermagem que atua nos Centros de Atenção Psicossocial de Maceió-AL e, assim, trouxe questões referentes às informações sociodemográficas, a formação desses profissionais, aspectos relacionados ao trabalho nos serviços e as principais atividades desenvolvidas.

Percebeu-se que a maioria dos profissionais era composta de mulheres e que grande parte são servidores públicos efetivos e que possuem sua formação há mais de cinco anos. A maioria está trabalhando nos CAPS há mais de cinco anos e nunca recebeu treinamento para o trabalho nos serviços, o que pode comprometer a efetivação do processo de reabilitação psicossocial. Todos trabalham 
em regime de 30 horas semanais, independente dos CAPS em que atuam.

As atividades mais desempenhadas pelas equipes de enfermagem são: administração de medicamentos, curativos simples, verificação dos sinais vitais, exame de glicemia capilar (HGT), visitas domiciliares e participação dos grupos e oficinas terapêuticas, e exclusivamente aos enfermeiros: supervisão da equipe de enfermagem, participação e construção dos grupos terapêuticos, construção do PTS dos usuários, consulta de enfermagem, acolhimento e visitas domiciliares.

Percebeu-se que os profissionais de enfermagem são fundamentais para a realização das ações nos Centros de Atenção Psicossocial de Maceió, haja vista que algumas das atividades desempenhadas são atribuições específicas do processo de trabalho destes. Não é possível imaginar os serviços de saúde mental sem a colaboração desses profissionais, desde as questões administrativas até mesmo no processo de reabilitação psicossocial das pessoas em sofrimento mental que são assistidas nesses dispositivos.

\section{REFERÊNCIAS}

1. Anjos EA, Nascimento YCML. O enfermeiro no caminho do cuidado à saúde mental. 1th ed. Maceió: EDUFAL; 2015.

2. Rocha RM. Enfermagem em saúde mental. 2th ed. Rio de Janeiro: Senac Nacional; 2014.
3. Silva MS, Machado PAT, Nascimento RS, Oliveira TS, Silva TF, Batista EC. SILVA, M. S. et al. A enfermagem no campo da saúde mental: uma breve discussão teórica. Revista Amazônia Science \& Health. 2017;2(2):40-46.

4. Botti NCL. Uma viagem na história da enfermagem psiquiátrica no início do século XX. Esc. Anna Nery. 2006;10(4):725-729.

5. Ribeiro MC. Trabalhadores dos Centros de Atenção Psicossocial de Alagoas, Brasil: interstícios de uma nova prática. InterfaceComunicação, Saúde, Educação. 2015;19(52):95-108.

6. Ministério da Saúde (BR). Portaria $N^{\circ}$ 3.588. Altera as Portarias de Consolidação

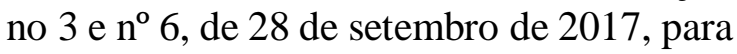
dispor sobre a Rede de Atenção Psicossocial, e dá outras providências. 2017.

7. Silva JVS, Brandão TM, Oliveira KCPN. Ações e Atividade desenvolvidas pela enfermagem no Centro de Atenção Psicossocial: Revisão Integrativa. Rev Enferm Atenção Saúde [Online]. 2018;7(3):137-149.

8. Brandão TM, Nascimento YCML, Brêda MZ, Albuquerque MCS, Albuquerque RS. A práxis do enfermeiro na atenção psicossocial: vulnerabilidades e potencialidades presentes. Rev. enferm. UFPE on line. 2016;10(6):4766-4777.

9. Lima-Costa MF, Barreto SM. Tipos de estudos epidemiológicos: conceitos básicos e aplicações na área do envelhecimento. Epidemiologia e serviços de saúde. 2003;12(4):189-201.

10. Vieira S. Introdução à bioestatística. 5 th ed. Brasil: Elsevier; 2015.

11. Machado MH (Org.). Convênio: FIOCRUZ/COFEN. Relatório final da Pesquisa Perfil da Enfermagem no Brasil Rio de Janeiro: FIOCRUZ; 2015.

12. Machado MH, Vieira ALS, Oliveira E. Construindo o perfil da enfermagem. Enfermagem em foco. 2012;3(3):119-122.

13. Lopes AG, Santos G, Ramos MM, Meira VF, Maia LFS. O desafio da 
educação permanente no trabalho da enfermagem. Revista Remecs. 2016;1(1):13-23.

14. Ventura CAA, Moll MF, Araújo AS, Jorge MS. A enfermagem e as dimensões organizacionais de dois centros de atenção psicossocial. Cienc Cuid Saude. 2015;14(2): 97-104.

15. Brasil. Decreto $N^{\circ} 7.508$, de 28 de junho de 2011. Regulamenta a Lei ${ }^{0} 8.080$, de 19 de setembro de 1990, para dispor sobre a organização do Sistema Único de Saúde - SUS, o planejamento da saúde, a assistência à saúde e a articulação interfederativa, e dá outras providências; 2011.

16. Muniz MP, Tavares CMM, Abrahão AL, Souza AC. A assistência de enfermagem em tempos de reforma psiquiátrica. Revista Portuguesa de Enfermagem de Saúde Mental. 2015;(13):61-65.

17. Esperidião E, Silva NS, Caixeta CC, Rodrigues J. A Enfermagem Psiquiátrica, a ABEn e o Departamento Científico de Enfermagem Psiquiátrica e Saúde Mental: avanços e desafios. Rev. bras. enferm. 2013;66(spe.):171-176.

18. Ribeiro MC, Chaves JB,; Barros AC, Correia MS, Lessa RO, Tavares LN. O trabalho nos centros de atenção psicossocial em uma capital do nordeste: limites e desafioS. Rev. baiana saúde pública. 2017;40(3):599:615.

19. Esperidião E, Cruz MFR, Silva G. A. Perfil e atuação dos enfermeiros da rede especializada em saúde mental de GoiâniaGO. 2011;13(3):493-501.

20. Sousa YG, Medeiros SM. Oficinas terapêuticas resinificando o cuidar de enfermagem nos centros de atenção psicossocial. Enfermagem Revista. 2018;20(1):23-30.

RECEBIDO: 06/11/18

APROVADO: 01/04/19

PUBLICADO: 07/19 